\title{
¿Paridad equivale a igualdad? Eficiencia de la producción científica de las investigadoras venezolanas
}

\author{
Preiddy Efraín García \\ Carlos García Zorita \\ Elías Sanz Casado *
}

Artículo recibido:

30 de julio de 2013.

Artículo aceptado:

24 de septiembre de 2013.

\section{Resumen}

Las políticas de igualdad de género promovidas desde distintos países y regiones han supuesto un avance importante a la hora de facilitar la plena incorporación de la mujer en la actividad científica. Esto ha dado lugar a la realización de numerosos estudios tendentes a determinar la posible existencia de diferencias en la actividad científica realizada por hombres y mujeres. El objetivo de este trabajo se ha dirigido a conocer si existen o no diferencias de productividad en determinadas áreas científicas en función del género de los investigadores, y la relación entre la posición en la carrera profesional de hombres y mujeres y su producción científica.

* Los tres autores pertenecen a la Universidad Carlos III de Madrid, Unidad Asociada IEDCYT-LEMI, España. pegarcia@bib.uc3m.es; czorita@bib.uc3m.es; elias@bib. uc3m.es

INVESTIGACIÓN BIBLIOTECOLÓGICA, Vol. 28, Núm.62, enero/abril, 2014, México, ISSN: 0187-358X. pp. 101-122 
Para ello se ha realizado un análisis bibliométrico de los 6015 currículums vitae de los investigadores venezolanos incluidos en el Programa de Promoción del Investigador (PPI) hasta el año 2009. Entre los resultados hay que destacar la mayor presencia de la mujer en el sistema de investigación venezolano, una menor presencia de mujeres en las categorías profesionales superiores, una menor productividad de las mujeres, o la diferente eficiencia que muestran las mujeres en función de las áreas temáticas consideradas.

Palabras clave: Paridad de género; Igualdad de género; Bibliometría; Productividad científica; Investigadores venezolanos.

\section{Abstract}

Is parity the same as equality: Efficiency of scientific output of Venezuelan female researchers

Preiddy Efrain-García, Carlos García-Zorita and Elias Sanz-Casado

Gender equality policies currently in place in many countries have spurred significant progress in advancing women in the fields of science. Nonetheless, numerous of recent studies have sought to measure differences between the scientific activity of men and women. The aim of this paper is to ascertain possible differences in scientific productivity in certain scientific disciplines carried out by men versus women and to determine importance of professional standing with regard to these scientific outputs. To this end, a bibliometric analysis was conducted of the curricula vitae of 6015 Venezuelan researchers participating in the country's Researcher Promotion Program up to 2009. Findings show that the Venezuelan research system has more female than male participants, but fewer women in the highest professional echelons. Women also proved to be less productive, with performance varying significantly from field to field.

Keywords: Gender parity; Gender equality; Bibliometrics; Scientific productivity, Venezuelan researchers. 


\section{INTRODUCCIÓN}

$\mathrm{F}^{1}$ análisis de la actividad científica en función del género está dando lu- gar a numerosos estudios comparativos que tienen como finalidad conocer si existen o no diferencias en las características de la actividad científica que están realizando los investigadores de ambos sexos. Para ello los análisis bibliométricos están resultando de gran utilidad, al permitir analizar las características de la actividad investigadora de una manera objetiva, ver cómo está evolucionando esta actividad y comparar los resultados con los de otras áreas de conocimiento o regiones geográficas.

Uno de los aspectos que más ha interesado determinar de la actividad investigadora es si existen o no diferencias en la producción científica en función del género de los investigadores. En estudios realizados con esta finalidad Prozesky (2006) observó que las publicaciones de los investigadores masculinos en las instituciones universitarias de Sudáfrica casi duplicaban a las de las investigadoras más productivas. Otro de los resultados obtenidos en este estudio fue que los investigadores masculinos más productivos superaron con creces la productividad de sus colegas femeninas más productivas. Esta misma autora observó que se seguían patrones similares en otros países analizados, donde las mujeres investigadoras producen menos en términos de publicaciones científicas que los hombres investigadores (Prozesky, 2008).

Estudios con objetivos similares se realizaron en España en el área de Ciencia de Materiales del Consejo Superior de Investigaciones Científicas (CSIC) (Mauleón y Bordons, 2006). Entre los resultados del estudio habría que destacar la escasa presencia de las mujeres en las categorías más avanzadas de la carrera profesional, el menor número de documentos publicados por las investigadoras en revistas incluidas en el Science Citation Index (SCI/ Thomson Reuters), o el impacto similar que tienen las publicaciones realizadas tanto por hombres como por mujeres. Otro estudio posterior (Mauleón, Bordons y Oppenheim, 2008), también realizado con el fin de conocer la actividad científica y tecnológica de los investigadores del CSIC desde la perspectiva de género, mostró que el promedio de documentos publicados por los hombres (male scientists) en revistas incluidas en la Web of Science era ligeramente superior al publicado por mujeres en la mayoría de las áreas, pero las diferencias fueron significativas sólo en las áreas de Alimentación, donde las mujeres mostraban una mayor productividad, y en Ciencia de Materiales y Ciencias Agrarias, donde la mayor productividad se encontró en los hombres.

Con objetivos similares Abramo, D’Angelo y Caprasecca (2009) obtuvieron resultados muy parecidos en cuanto a mayor productividad de los científicos frente a las científicas en su análisis de las diferencias de género en el 
sistema académico italiano. Sin embargo, resultados distintos en cuanto a productividad científica fueron obtenidos por De Filippo, Sanz-Casado y Gómez (2009) ya que observaron que la productividad científica no mostraba diferencias significativas entre los investigadores de ambos sexos.

En este trabajo se ha querido conocer si en el caso de Venezuela la investigación que se realiza sigue patrones distintos en función del género de los investigadores. En Venezuela, a partir de la década de los noventa, se fueron introduciendo una serie de acciones con la finalidad de fomentar la implicación de los científicos en su labor investigadora y concretamente para incrementar las publicaciones de sus resultados de investigación. Con esta finalidad nace en 1990 la Fundación Venezolana para la Promoción del Investigador, la cual mediante su Programa de Promoción del Investigador (PPI) trata de darle un nuevo impulso al desarrollo científico. El origen del programa está en los esfuerzos llevados a cabo en la década de los ochenta para establecer un sistema de reconocimiento para los investigadores de la Universidad Central de Venezuela (ONCTI, 2007; Marcano y Phélan , 2009). En el decreto de creación de la Fundación Fondo del Sistema de Promoción del Investigador, el artículo 2 establece que la "Fundación tendrá como objeto prestar la asistencia económica necesaria para cubrir las obligaciones que sean requeridas con ocasión de la aplicación y desarrollo de los programas del PPI” (República de Venezuela, 1990).

El Programa de Promoción del Investigador es uno de los programas que ejecuta el Observatorio Nacional de Ciencia, Tecnología e Innovación (ONCTI), el cual tiene como objetivo contribuir a darle visibilidad a la actividad científica y tecnológica que realizan los investigadores radicados en Venezuela. Los investigadores son evaluados por pares constituidos en las comisiones de Áreas y clasificados en tres categorías: Candidato, Investigador y Emérito. A su vez, la categoría de investigador comprende cuatro niveles: I, II, III y IV (Marcano y Phélan, 2009).

Asimismo, dicho programa se encarga de llevar a cabo un registro permanente de los investigadores en el país, construyendo su propia herramienta para la captura y posterior almacenamiento de los datos aportados en el curriculum vitae (CV). Para los investigadores, el CV representa a la vez un registro de su logro científico y una obligación administrativa ante instancias superiores, por ello, y entre otras razones, los investigadores se ven incentivados a mantener su CV actualizado y fácilmente accesible.

En el ámbito de la comunicación científica, el CV es una de las pocas fuentes casi universales en cuanto a su disponibilidad y significado. Así pues, el $\mathrm{CV}$ se muestra como una interesante fuente de datos para la evaluación de los investigadores y de su actividad científica (Sandström, 2009). Sin embargo, 
su uso como tal se inicia en la década de los noventa, con unos pocos estudios sobre el uso del CV como fuente de información suplementaria (Cañibano, Otamendi y Andújar, 2008; Martín-Sempere y Rey-Rocha, 2003; Gaughan y Bozeman, 2002).

El objetivo de este trabajo es determinar si existen diferencias de género en determinadas características de la actividad científica realizada por los investigadores e investigadoras venezolanos, a partir de los datos que aportan en sus CV. Algunas de las principales cuestiones que se pretende contestar son: a) ¿existen diferencias de productividad en cada una de las áreas consideradas en función del género de los investigadores?; b) ¿Existe relación entre la posición en la carrera profesional de hombres y mujeres y su producción científica?

Asimismo es importante señalar que sólo se analiza la actividad científica de los investigadores que se mantenían activos en el momento de la toma de datos, y por tanto, se pueden poner en relación directa los resultados de la actividad científica (output) con la población que los genera (inputs). Este hecho diferencia este trabajo de otros estudios en los que se asocia la producción científica con toda la población investigadora, sea ésta la responsable o no de dicha producción (Abramo, D’Angelo y Caprasecca, 2009).

\section{MATERIAL Y MÉTODOS}

Para la elaboración del presente estudio se obtuvieron los datos en el año 2010 correspondientes a los CV de los investigadores venezolanos adscritos al PPI. Estos datos estaban accesibles a través de la página web del Programa de Promoción del Investigador (García González, 2010). Los datos de los investigadores se agruparon por el género. En total se obtuvieron $6015 \mathrm{CV}$ con producción científica registrada en el sistema hasta el año 2009. En este análisis se utilizan como inputs el número de investigadores e investigadoras implicados en cada una de las disciplinas académicas en las que se clasificaron, y como outputs, la producción científica por género, medida por las publicaciones en revistas nacionales e internacionales en cada una de las áreas.

Para los análisis descriptivos se utilizan funciones del 'base package' del software estadístico libre R (R Development Core Team, 2010); para el cálculo de los índices de Gini se utiliza el paquete 'ineq' (Zeileis, 2012) que implementa una función para su cálculo basada en la formulación de Allison (1978), compatible con las formulaciones utilizadas en el ámbito de la Informetría (Rousseau, 1998, 2000). Respecto al número de investigadores y sus publicaciones, se elaboran tablas de contingencia en las que se combinan las variables género y área temática. 
Por otro lado, en este trabajo los investigadores venezolanos se han agrupado en tres categorías: Candidato (Ca), Investigadores de Nivel I (L_1) e Investigadores clasificados en los niveles superiores del PPI (Up_L). Estos tres niveles de agrupación se combinaron con las variables género y disciplina académica, tanto para el número de investigadores (input) como para el número de publicaciones (output).

Para un análisis exploratorio y visual de tablas de contingencia se han empleado gráficos de mosaico utilizando el paquete 'vcd' (Meyer et al., 2012). Un gráfico de mosaico es una representación gráfica constituida por una especie de "baldosas" cada una de las cuales es proporcional en sus dimensiones, ancho y alto, a cada una de las celdas (frecuencias observadas) de la correspondiente tabla de contingencia (Meyer et al., 2006).

La eficiencia de género se ha medido utilizando el Gender Parity Index o Índice de paridad de género (GPI) (UNESCO, 1997), que permite determinar la integración de la mujer en las tareas de investigación. Éste se calcula para cada una de las disciplinas académicas estudiadas, tanto a nivel de input como de output. Así pues, se calcula por un lado el GPI en relación con los recursos empleados en investigación (GPI_r), que vendrá dado por la relación entre el número de mujeres investigadoras frente al de hombres. Los valores superiores a la unidad indican una mayor presencia femenina. Por otra parte, se calcula el GPI en relación con la distribución por género de las publicaciones científicas (GPI_p), y se mide por la relación entre el número de publicaciones realizadas por mujeres investigadoras frente al de publicaciones de los hombres. Valores por encima de la unidad muestran una mayor productividad de las mujeres investigadoras.

La Tasa de eficiencia de género o Gender Success Rate (GSR), se define como la capacidad que muestran los investigadores de un determinado género para ser más eficientes en su actividad científica. Se calcula poniendo en relación los índices de paridad GPI_i y GPI_p. Valores por encima de la unidad se corresponderían con una mayor eficiencia femenina.

\section{Resultados Y DisCUSión}

\section{Distribuciones del número de investigadores ( $r$ ) y del número de publicaciones (p) por disciplina académica}

El número total de investigadores venezolanos que se analizaron en este estudio fue de 6015; de ellos 2823 son hombres y 3192 mujeres, todos ellos adscritos al PPI. En la Tabla 1 se presenta su distribución por género y área 
científica, así como la distribución, igualmente por género y disciplina académica, del número de publicaciones reflejadas por estos investigadores en sus CV. Los datos están ordenados por número decreciente del total de investigadores por área.

Cuando se analizan los datos de la Tabla 1 se observa que globalmente el porcentaje de mujeres es un $6.13 \%$ superior al de hombres. Este dato está en consonancia con la incorporación de la mujer en la educación superior en Venezuela, que pasó del 43 \% en 1979, al 59 \% en 2005 (Delgado de Smith y Rojas, 2009). Esta feminización de la investigación venezolana, especialmente en el área de Ciencias de la Vida, ha sido también reportada en un trabajo reciente por Caputo, Requena y Vargas (2012). En España se ha observado una evolución similar, aunque partiendo de porcentajes más bajos, ya que en 1972 el porcentaje de docentes universitarias era de un $15 \%$ y en los años noventa este porcentaje se incrementó hasta el $29 \%$ (Pérez Sedeño et al., 2003). Sin embargo, en publicaciones son los hombres los que muestran un porcentaje global más elevado $(8.35 \%)$. En trabajos realizados con investigadores españoles también se ha observado una mayor actividad publicadora por parte de los hombres (Mauleón y Bordons, 2006). Cuando se analiza cada área este porcentaje varía de unas a otras pero el área Otros (Other) no se ha considerado en el análisis al incluirse en ella varias áreas distintas con escasa presencia tanto en investigadores como en publicaciones). Por ejemplo, en las disciplinas de Ciencias Médicas (Med Sci), Pediatría (Ped), Economía (Econ), Lingüística (Ling), Derecho (Law), Psicología (Psych) y Ética (Ethics), la presencia de mujeres respecto a la de hombres es superior al $60 \%$. Sin embargo, cuando se comparan estos porcentajes con los de publicaciones realizadas por las mujeres en esas mismas áreas se observa que todos los valores son inferiores respecto a su presencia en cada una de ellas. Cabe destacar el caso de Psicología (Psych) donde el porcentaje es prácticamente similar, $74.5 \%$ y $74.28 \%$ respectivamente.

Cuando se hace la misma comparación con los hombres, las áreas donde estos tienen porcentajes de presencia superiores al $60 \%$ son: Física (Phys), Matemáticas (Math), Ciencias de la Tierra (Earth Sci), Filosofía (Philo) y Astronomía y Astrofísica (Astro). Cuando se comparan estos valores con los porcentajes de sus publicaciones en estas áreas, se observa que todos los valores son más elevados excepto en Filosofía, que es ligeramente inferior (56.32\%). 
Tabla 1. Distribuciones de la presencia de investigadores en las publicaciones por disciplina académica y por género

\begin{tabular}{|l|c|c|c|c|c|c|c|c|c|c|}
\hline & \multicolumn{3}{|c|}{ Número de investigadores (r) } & \multicolumn{4}{c|}{ Número de Publicaciones (p) } \\
\hline Áreas & Hombre & Mujer & Total & $\begin{array}{c}\text { Hombre } \\
(\%)\end{array}$ & $\begin{array}{c}\text { Mujer } \\
(\%)\end{array}$ & Hombre & Mujer & Total & $\begin{array}{c}\text { Hombre } \\
(\%)\end{array}$ & $\begin{array}{c}\text { Mujer } \\
(\%)\end{array}$ \\
\hline Life Sci & 377 & 473 & 850 & 44.4 & 55.6 & 8062 & 6432 & 14494 & 55.62 & 44.38 \\
\hline Tech Sci & 386 & 345 & 731 & 52.8 & 47.2 & 5993 & 5430 & 11423 & 52.46 & 47.54 \\
\hline Med Sci & 226 & 436 & 662 & 34.1 & 65.9 & 4005 & 5818 & 9823 & 40.77 & 59.23 \\
\hline Agr Sci & 341 & 289 & 630 & 54.1 & 45.9 & 6144 & 4186 & 10330 & 59.48 & 40.52 \\
\hline Ped & 155 & 339 & 494 & 31.4 & 68.6 & 1796 & 3241 & 5037 & 35.66 & 64.34 \\
\hline Chem & 240 & 203 & 443 & 54.2 & 45.8 & 5610 & 2995 & 8605 & 65.19 & 34.81 \\
\hline Econ & 106 & 164 & 270 & 39.3 & 60.7 & 1087 & 1557 & 2644 & 41.11 & 58.89 \\
\hline Phys & 205 & 43 & 248 & 82.7 & 17.3 & 3958 & 762 & 4720 & 83.86 & 16.14 \\
\hline Sociol & 94 & 136 & 230 & 40.9 & 59.1 & 2096 & 2025 & 4121 & 50.86 & 49.14 \\
\hline Math & 151 & 74 & 225 & 67.1 & 32.9 & 2174 & 881 & 3055 & 71.16 & 28.84 \\
\hline Arts & 67 & 97 & 164 & 40.9 & 59.1 & 1384 & 1452 & 2836 & 48.8 & 51.20 \\
\hline Earth Sci & 101 & 60 & 161 & 62.7 & 37.3 & 2248 & 979 & 3227 & 69.66 & 30.34 \\
\hline Ling & 38 & 102 & 140 & 27.1 & 72.9 & 672 & 1512 & 2184 & 30.77 & 69.23 \\
\hline Hist & 60 & 63 & 123 & 48.8 & 51.2 & 1328 & 1222 & 2550 & 52.08 & 47.92 \\
\hline Polit & 57 & 60 & 117 & 48.7 & 51.3 & 1317 & 1003 & 2320 & 56.77 & 43.23 \\
\hline Law & 35 & 77 & 112 & 31.2 & 68.8 & 554 & 891 & 1445 & 38.34 & 61.66 \\
\hline Psych & 28 & 82 & 110 & 25.5 & 74.5 & 386 & 1115 & 1501 & 25.72 & 74.28 \\
\hline Philo & 45 & 27 & 72 & 62.5 & 37.5 & 722 & 560 & 1282 & 56.32 & 43.68 \\
\hline Anthro & 34 & 27 & 61 & 55.7 & 44.3 & 690 & 566 & 1256 & 54.94 & 45.06 \\
\hline Geo & 20 & 21 & 41 & 48.8 & 51.2 & 259 & 232 & 491 & 52.75 & 47.25 \\
\hline Astro & 15 & 5 & 20 & 75 & 25.0 & 491 & 110 & 601 & 81.7 & 18.30 \\
\hline Ethics & 7 & 11 & 18 & 38.9 & 61.1 & 69 & 101 & 170 & 40.59 & 59.41 \\
\hline Other & 35 & 58 & 93 & 37.6 & 62.4 & 207 & 286 & 493 & 41.99 & 58.01 \\
\hline Total & 2823 & 3192 & 6015 & & & 51252 & 43356 & 94608 & & \\
\hline
\end{tabular}

En la Figura 1 se muestra el diagrama de caja (Box-Plot) correspondiente a los datos de la Tabla 1. Se observa que el promedio de investigadoras es algo superior al de sus compañeros masculinos, siendo las Ciencias de la Vida (Life Sci) y las Ciencias Médicas (Med Sci) las dos áreas que presentan un número atípico de investigadoras. Por otro lado, la media del número de publicaciones es menor entre las investigadoras con un promedio de 1885 documentos, frente a los 2228 de los investigadores varones. El número de publicaciones en el área de Ciencias de la Vida (Life Sci) se comporta de modo diferenciado tanto en hombres como en mujeres. Para éstas, además, muestran un comportamiento atípico las áreas de Ciencias Tecnológicas (Tech Sci) y las de Ciencias de la Vida (Med Sci).

Ambas distribuciones no presentan diferencias significativas respecto al género de los investigadores. Los test de análisis de varianza para el número 
de investigadores $\left(\mathrm{F}_{2,44}=0.176, p\right.$-value: 0.6768$)$ y para el número de publicaciones $\left(\mathrm{F}_{2,44}=0.3099, p\right.$-value $\left.=0.5805\right)$ así lo demuestran.

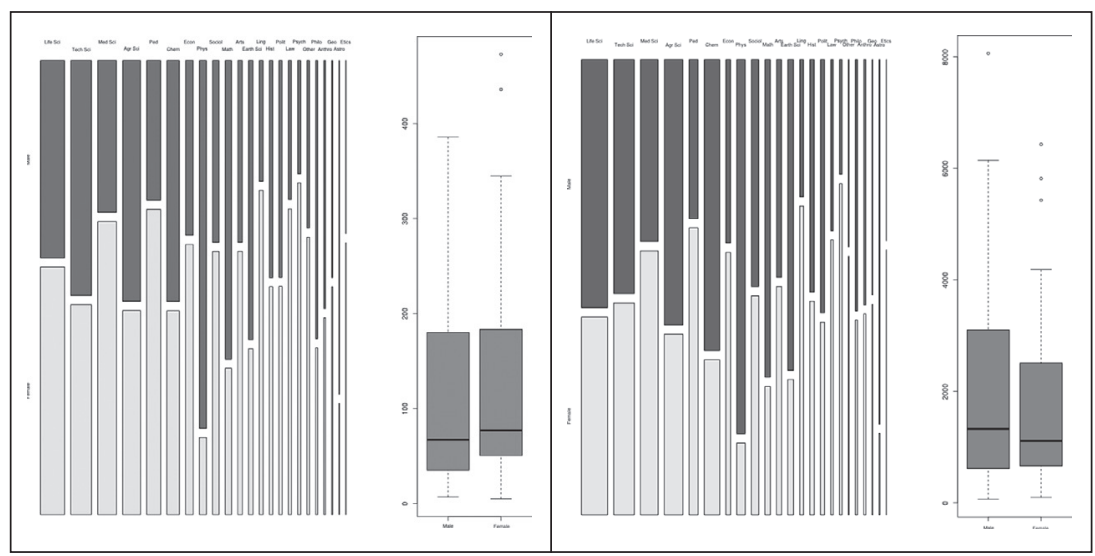

Figura 1. Gráficos de mosaico y diagramas de caja de la distribución de investigadores y de sus publicaciones por área y género

El análisis de los datos por género del número de investigadores y publicaciones frente al nivel de la categoría profesional se muestra en la Tabla 2. En dicha tabla se observa que de manera general las mujeres investigadoras tienen una mayor presencia en los niveles más bajos de su categoría profesional, como son el L_1 y el Candidato (Ca), ya que representan el $22.1 \%$ y el $18.57 \%$ del total de investigadores, mientras que los hombres tienen una mayor presencia en los niveles superiores (Up_L) con el $16.37 \%$. Resultados similares han sido obtenidos en otros trabajos en los que se ha observado que las mujeres investigadoras tenían mayor presencia que los hombres en los niveles más bajos de su categoría profesional, y menor presencia en los más altos (Abramo, D’Angelo y Caprasecca, 2009; Mauleón y Bordons, 2006; Mauleón, Bordons y Oppenheim, 2008; Caputo, Requena y Vargas, 2012). En cuanto a la producción científica en el caso de las mujeres investigadoras, ésta es superior en los niveles más altos de su categoría (Up_L), siendo responsables del $22.6 \%$ del total de publicaciones considerado. Los hombres también tienen mayor producción científica en el nivel más alto (Up_L), ya que realizan el $34.79 \%$ de sus publicaciones, y lo son en una proporción mucho más elevada que en el caso de las mujeres.

Cuando se analiza en cada una de las áreas consideradas la presencia de mujeres y su producción científica en función de su categoría profesional, se observa que en la primera etapa de su carrera (Ca) las mujeres tienen un mayor número en 15 de las 22 áreas analizadas, mientras que los hombres lo tienen en 7 de ellas (Ciencias Tecnológicas (Tech Sci), Física (Phys), Matemáticas 


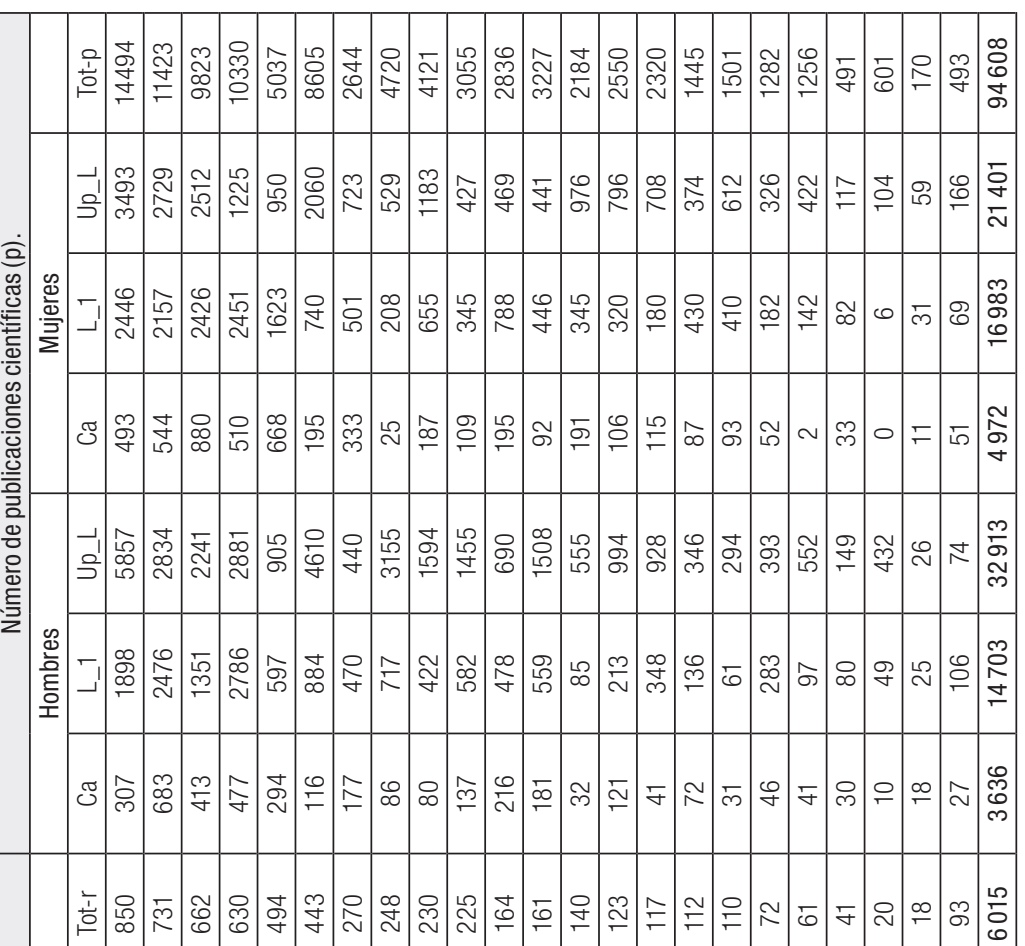

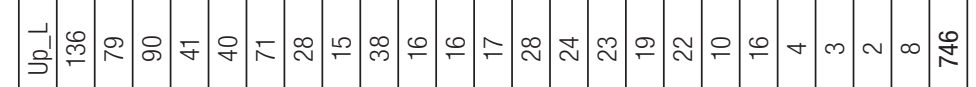

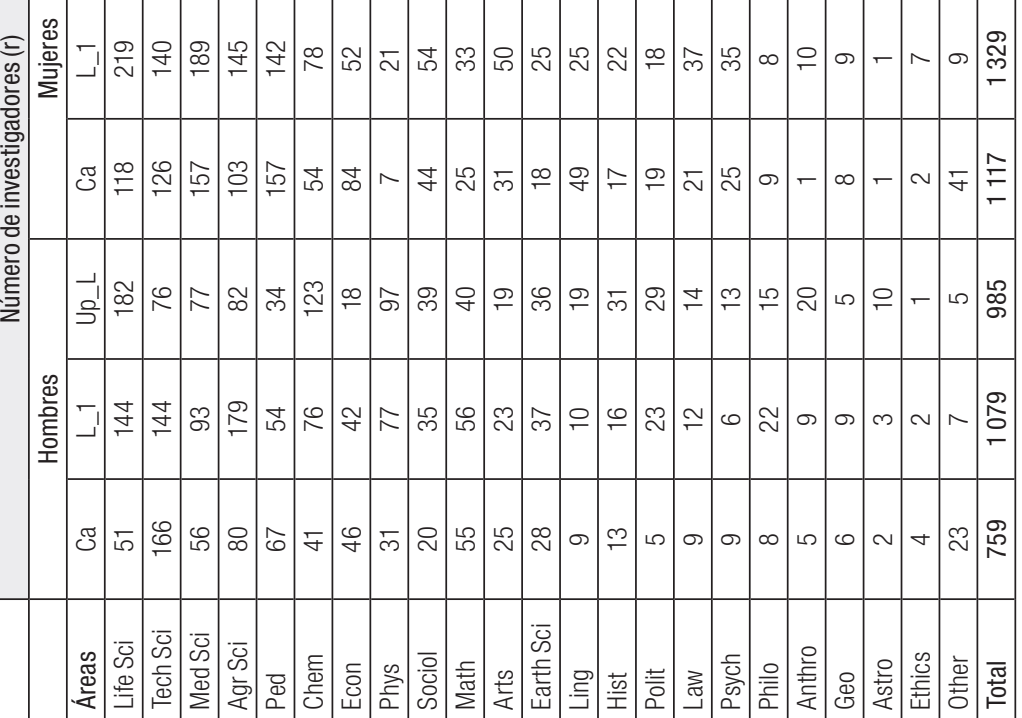


(Math), Ciencias de la Tierra (Earth Sci), Antropología (Anthro), Astrofísica (Astro) y Ética (Ethics)). Sin embargo, el número de publicaciones de las mujeres en este nivel de su categoría es inferior al de los hombres en 9 áreas, de las 7 que se han mencionado y en la de Artes e Historia (Arts).

En el siguiente nivel de la categoría profesional (L_1) disminuyen las áreas en las que las mujeres son más numerosas que los hombres, puesto que están presentes en 13 de las 22 áreas analizadas. En cuanto al número de publicaciones, la mayoría de las áreas en las que las mujeres cuentan con una mayor presencia son también aquellas en las que publican un mayor número de documentos, excepto en Química (Chem) donde los hombres investigadores publican 144 documentos más que las mujeres, a pesar de contar con 2 investigadores menos.

Respecto a los niveles más altos de la categoría ( $U_{\text {_ }}$ L), el número de áreas con un número mayor de mujeres respecto al de hombres disminuye hasta 8. Cuando se analizan las áreas donde las mujeres publican un mayor número de documentos, se observa que 7 de esas áreas coinciden con aquellas donde su presencia es más numerosa. En Ciencias Tecnológicas (Tech Sci), a pesar de ser mayor el número de mujeres que de hombres éstos publican más que las mujeres.

Las diferencias observadas en relación con el número de investigadores mostrados en la Tabla 2 no son estadísticamente significativas ni respecto al género de los investigadores $\left(\mathrm{F}_{1,136}=0.4381, p\right.$-value $\left.=0.5092\right)$ ni en cuanto a su categoría profesional $\left(\mathrm{F}_{2,135}=1.236\right.$, p-value $\left.=0.2939\right)$. En cambio, para el número de publicaciones las diferencias son significativas para la categoría profesional $\left(\mathrm{F}_{2,135}=14.84\right.$, p-value $\left.<0.05\right)$, pero no lo son para el género $\left(\mathrm{F}_{1,136}=0.4894\right.$, p-value $\left.=0.4854\right)$.

Para determinar si el número de investigadores y el de sus publicaciones se reparten de un modo equitativo entre las distintas áreas temáticas se han calculado para cada una de estas distribuciones los índices de Gini, y se han dibujado sus correspondientes curvas de Lorentz. Ambos resultados se muestran en la Tabla 3 y en la Figura 2 (página siguiente).

Como puede observarse los niveles de concentración no son excesivamente altos. El comportamiento global tanto en el número de investigadores (0.4824) como en el de su producción científica (0.4916) son muy similares. Se entiende que hay una mayor homogeneidad entre los investigadores del nivel más alto (Up_L), y entre éstos, las distribuciones son algo más uniformes en el caso de las mujeres investigadoras que en el de sus colegas masculinos. En relación con los otros dos niveles considerados (Ca y L_1), las distribuciones respecto al número de publicaciones se muestran algo más concentradas que las del número de investigadores. 
Tabla 3. Índices de Gini: Distribuciones por área y por género

\begin{tabular}{|l|c|c|c|c|c|c|}
\hline & \multicolumn{3}{|c|}{$\begin{array}{c}\text { Número de investigadores } \\
(\mathrm{r})\end{array}$} & \multicolumn{3}{c|}{$\begin{array}{c}\text { Número de publicaciones } \\
\text { científicas (p) }\end{array}$} \\
\hline & $\mathrm{Ca}$ & $\mathrm{L}_{-} 1$ & UP_L & $\mathrm{Ca}$ & $\mathrm{L}_{-} 1$ & UP_L \\
\hline Hombres & 0.5207 & 0.5386 & 0.5028 & 0.5328 & 0.5704 & 0.5275 \\
\hline Mujeres & 0.5313 & 0.5406 & 0.4781 & 0.5521 & 0.5544 & 0.4815 \\
\hline Total & \multicolumn{3}{|c|}{0.4824} & \multicolumn{3}{c|}{0.4916} \\
\hline
\end{tabular}

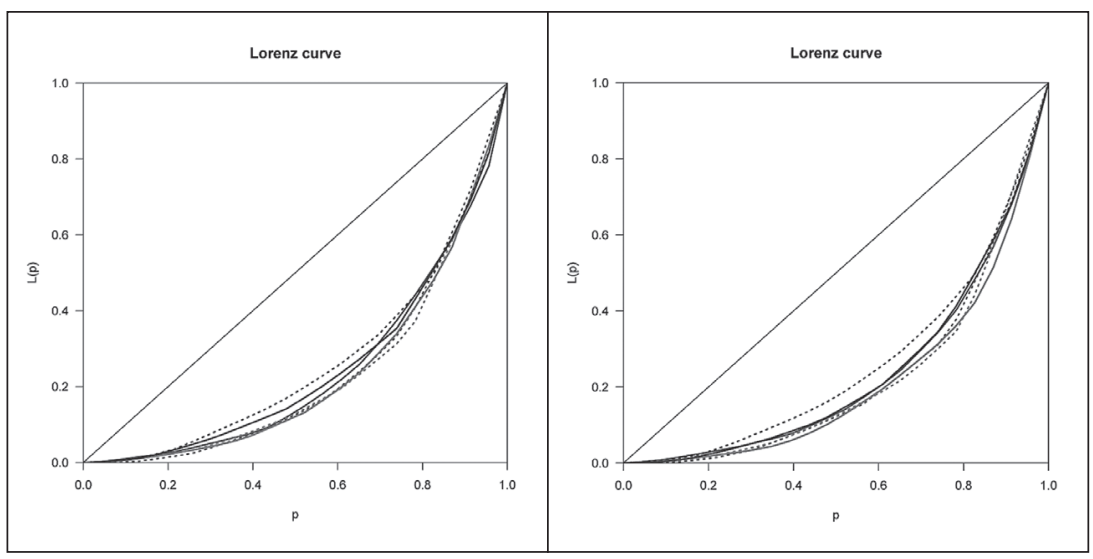

Figura 2. Curvas de Lorenz. Izq.: por número de investigadores. Der.: por número de publicaciones científicas. Hombres: líneas continuas; Mujeres: líneas de puntos.

\section{Índices de paridad de género (Gender Parity Indexes, GPI)}

En la Tabla 4 se muestran los índices de paridad de género calculados en cada una de las categorías profesionales y para cada una de las áreas temáticas analizadas. Cuando se consideran todas las áreas en general (como en los casos anteriores, la categoría Otros (Other) no se ha considerado al incluirse en ella varias áreas) se observa que en relación al número total de investigadores adscritos a cada una de las áreas, en 8 de ellas la presencia de la mujer es notablemente superior a la de los hombres. Psicología (Psych, 2.929), Lingüística (Ling, 2.684), Derecho (Law, 2.200), Pediatría (Ped, 2.187), Ciencias Médicas (Med Sci, 1.929), Economía (Econ, 1.547), Sociología (Sociol, 1.447) y Ciencias de la Vida (Life Sci, 1.255). Sin embargo, cuando se analiza la productividad en estas áreas en todas ellas se encuentran valores inferiores a los obtenidos por las mujeres respecto a su número, y en 2 de ellas, Sociología (Sociol, 0.966) y Ciencias de la Vida (Life Sci, 0.798), la productividad de los hombres es superior a pesar de su inferioridad numérica. 
Desde el punto de vista de la paridad por niveles de adscripción de los investigadores, en las tres categorías profesionales los resultados varían en función del área temática considerada, siendo estas diferencias significativas $\left(F_{2,66}=3.234\right.$, p-value $\left.<0.05\right)$. En términos generales se puede afirmar que los resultados obtenidos en este trabajo mostrarían que el peso de la mujer disminuye cuando se eleva el nivel de su carrera investigadora.

El promedio del valor del GPI ponderado por el número de investigadores en cada una de las áreas temáticas muestra un valor de 1.317 para el total de la población, algo superior a la proporción real de mujeres adscritas al sistema $(3192 / 2823=1.131)$. Esto es debido a la fuerte presencia de las mujeres en las áreas que más recursos humanos dedican a la investigación, como son Ciencias de la Vida (Life Sci), Ciencias Médicas (Med Sci) o Pediatría (Ped). De igual modo, se observa que a nivel agregado la presencia de la mujer disminuye según sea el nivel de los investigadores, quedando el nivel más alto por debajo de la paridad, puesto que los valores pasan de 1.80 en el primer nivel (Ca), a 1.47 en el segundo nivel (L_1) y a 0.83 en los niveles finales (Up_L)

Un comportamiento ligeramente diferente se observa cuando analizamos la distribución de la productividad científica de los hombres y mujeres adscritos al PPI, en función del género y del área temática. En términos agregados, medidos por la media ponderada por el número de publicaciones, globalmente se obtiene un GPI ponderado de 0.951 , que señala por un lado paridad global en el número de publicaciones, pero que al ser inferior al calculado para los recursos indica una pérdida de eficiencia en la productividad de las mujeres. Se observa así una relación similar a la ya obtenida para el número de hombres investigadores, en relación con la productividad femenina en función del nivel de la categoría profesional. Esta productividad disminuye desde los estados iniciales de candidato $(\mathrm{Ca})$, en los que la productividad femenina es $62.4 \%$ superior a la de sus compañeros masculinos, hasta valores de productividad de los investigadores de mayor nivel de adscripción (Up_L), en los que la productividad se decanta claramente hacia los hombres (0.734). En el nivel intermedio (L_1) las mujeres investigadoras pierden algo de productividad, pero en términos agregados se mantienen en un $37.6 \%$ por encima de la de los hombres. Esta menor productividad entre las investigadoras a medida que suben en su categoría profesional se mantiene en la mayoría de las áreas temáticas consideradas, a excepción, nuevamente, de las Ciencias Tecnológicas (Tech Sci), en las que consecuentemente con el comportamiento ya descrito desde el punto de vista de la presencia femenina, su productividad aumenta con su nivel profesional. Las diferencias observadas de los índices de paridad entre las áreas temáticas no son 
estadísticamente achacables al nivel de la categoría profesional de los investigadores $\left(\mathrm{F}_{2,66}=1.805\right.$, $p$-value $\left.=0.1725\right)$.

Tabla 4. GPI (índice de paridad de género) del número de investigadores y el número de publicaciones, por área de conocimiento y según la categoría del PPI (Programa de Promoción del Investigador).

\begin{tabular}{|l|c|c|c|c|c|c|c|c|}
\hline & \multicolumn{3}{|c|}{ Gúmero de investigadores $(r)$} & \multicolumn{3}{c|}{ Número de publicaciones científicas ( $p$ ) } \\
\hline & \multicolumn{5}{|c|}{ GPI-r } & \multicolumn{5}{c|}{ GPI- } \\
\hline Áreas & Ca & L_1 & Up_L & Total & Ca & L_1 & Up_L & Total \\
\hline Life Sci & 2.314 & 1.521 & 0.736 & 1.255 & 1.606 & 1.289 & 0.596 & 0.798 \\
\hline Tech Sci & 0.759 & 0.972 & 1.039 & 0.894 & 0.796 & 0.871 & 0.963 & 0.906 \\
\hline Med Sci & 2.804 & 2.032 & 1.156 & 1.929 & 2.131 & 1.796 & 1.121 & 1.453 \\
\hline Agr Sci & 1.288 & 0.81 & 0.5 & 0.848 & 1.069 & 0.88 & 0.425 & 0.681 \\
\hline Ped & 2.343 & 2.63 & 1.176 & 2.187 & 2.272 & 2.719 & 1.05 & 1.805 \\
\hline Chem & 1.317 & 1.026 & 0.577 & 0.846 & 1.681 & 0.837 & 0.447 & 0.534 \\
\hline Econ & 1.826 & 1.238 & 1.556 & 1.547 & 1.881 & 1.066 & 1.643 & 1.432 \\
\hline Phys & 0.226 & 0.273 & 0.144 & 0.21 & 0.291 & 0.29 & 0.168 & 0.193 \\
\hline Sociol & 2.2 & 1.543 & 0.974 & 1.447 & 2.338 & 1.552 & 0.742 & 0.966 \\
\hline Math & 0.455 & 0.589 & 0.4 & 0.49 & 0.796 & 0.593 & 0.293 & 0.405 \\
\hline Arts & 1.24 & 2.174 & 0.842 & 1.448 & 0.903 & 1.649 & 0.68 & 1.049 \\
\hline Earth Sci & 0.643 & 0.676 & 0.472 & 0.594 & 0.508 & 0.798 & 0.292 & 0.435 \\
\hline Ling & 5.444 & 2.5 & 1.474 & 2.684 & 5.969 & 4.059 & 1.759 & 2.25 \\
\hline Hist & 1.308 & 1.375 & 0.742 & 1.05 & 0.876 & 1.502 & 0.801 & 0.92 \\
\hline Polit & 3.8 & 0.783 & 0.793 & 1.053 & 2.805 & 0.517 & 0.763 & 0.762 \\
\hline Law & 2.333 & 3.083 & 1.286 & 2.2 & 1.208 & 3.162 & 1.081 & 1.608 \\
\hline Psych & 2.778 & 5.833 & 1.692 & 2.929 & 3 & 6.721 & 2.082 & 2.889 \\
\hline Philo & 1.125 & 0.364 & 0.667 & 0.6 & 1.13 & 0.643 & 0.83 & 0.776 \\
\hline Anthro & 0.2 & 1.111 & 0.8 & 0.794 & 0.049 & 1.464 & 0.764 & 0.82 \\
\hline Geo & 1.333 & 1 & 0.8 & 1.05 & 1.1 & 1.025 & 0.785 & 0.896 \\
\hline Astro & 0.5 & 0.333 & 0.3 & 0.333 & 0 & 0.122 & 0.241 & 0.224 \\
\hline Ethics & 0.5 & 3.5 & 2 & 1.571 & 0.611 & 1.24 & 2.269 & 1.464 \\
\hline Other & 1.783 & 1.286 & 1.6 & 1.657 & 1.889 & 0.651 & 2.243 & 2.383 \\
\hline Media ponderada & 1.805 & 1.469 & 0.829 & 1.317 & 1.624 & 1.376 & 0.734 & 0.951 \\
\hline & & & & & & & &
\end{tabular}

\section{Tasa de eficiencia del género (Gender Success Rate, GSR)}

En la Tabla 5 se presentan los resultados correspondientes a la eficiencia de la productividad de las mujeres investigadoras. Para ello, se muestran en dicha tabla los valores que representan la relación entre el output (número de publicaciones) y el input (número de investigadores) medido cada uno de ellos por su correspondiente valor de GPI. El valor de la Tasa de eficiencia de género determina el rendimiento observado en relación con el rendimiento esperado desde el punto de vista del género, medido por la participación 
de la mujer tanto en los resultados obtenidos (output) como en los recursos humanos empleados (input). Para entender mejor este indicador, habría que tener en cuenta que si en una determinada área la participación de la mujer representa un $80 \%$ de la de los hombres, en esa misma área (GPI-r $=0,80)$ se esperaría entonces que los resultados de su actividad científica, medida por el número de publicaciones científicas en sus CV sea similar a (GPI-p=0,80). $\mathrm{Si}$ el porcentaje de documentos publicados por las mujeres en relación al de los hombres es superior a ese porcentaje esperado, entonces el valor de GSR es mayor que 1 y podemos hablar del mayor éxito de las mujeres en esa área determinada en la que se muestran más productivas que los hombres.

Estas condiciones de éxito se dan sea cual sea la presencia de investigadores por género que se produzca en un área determinada; es decir, hay áreas en las que la presencia de las mujeres es mayor que la de los hombres y publican más que ellos (áreas marcadas con ** en la Tabla 5) y a pesar de ello los hombres son más eficientes, como es el caso de Med Sci (GSR=0.753) o Ped $(G S R=0.825)$. De igual manera, hay áreas en las que hay una mayor presencia de mujeres y sin embargo en términos de resultados presentan una menor proporción de publicaciones que los hombres (son las áreas marcadas con * en la Tabla 5). En este sentido, hay áreas como Ciencias de la Vida (Life Sci) en donde a pesar de que las mujeres en conjunto tienen una mayor presencia que los hombres son menos efectivas que éstos en el número de publicaciones $(G S R=0.636)$. Esto ocurre en cada uno de los tres niveles de su categoría profesional, por lo que llama la atención que en el nivel inicial de candidato (Ca) el valor de la GSR es de 0.694, y en el nivel L_1, el valor de GSR es de 0.847; es decir, que aun publicando más que los hombres, las mujeres lo hacen en una proporción menor de la esperada.

Además, se da la circunstancia de que en ningún caso se han observado áreas en las que las mujeres tengan una presencia numérica menor que los hombres y publiquen más que éstos, pero sí hay áreas en las que no se dan ninguna de las tres circunstancias mencionadas anteriormente (celdas sin marcar en la Tabla 5). En este contexto llama la atención el área de las Ciencias Tecnológicas (Tech Sci) donde las mujeres se muestran ligeramente más eficientes que los hombres (GSR=1.013), a pesar de que representan una menor fuerza investigadora (GPI- $\mathrm{r}=0.894$ ) y son menos productivas que ellos (GPI-p=0.906).

Por otra parte, en el área de Física (Phys) con una escasa presencia de mujeres (GPI-r $=0.21)$ y con una baja proporción de trabajos firmados por ellas (GPI-p=0.193), presentan una eficiencia de género cercana a la paridad (GSR=0.919). En Química (Chem), en el nivel candidato (Ca) las mujeres se muestran más eficientes que los hombres; sin embargo, cuando se analiza esto 
en su conjunto, son los hombres los que resultan más eficientes que las mujeres. Entre las ciencias sociales, Economía (Econ, GSR=0.926) y Psicología (Psych, GSR=0.986) las mujeres se muestran cercanas a la paridad de eficiencia de género, mientras que en la Sociología (Sociol) presentan un bajo índice de eficiencia $(G S R=0.668)$.

Tabla 5. Tasa de eficiencia de la productividad en mujeres y hombres por área de conocimiento y categoría profesional

\begin{tabular}{|c|c|c|c|c|}
\hline & \multicolumn{4}{|c|}{ Gender Success Rate (GSR) } \\
\hline Áreas & $\mathrm{Ca}$ & L_I & Up_L & Total \\
\hline Life Sci & $0.694^{\star \star}$ & $0.847^{\star \star}$ & 0.810 & $0.636^{*}$ \\
\hline Tech Sci & 1.049 & 0.896 & $0.927^{\star}$ & 1.013 \\
\hline Med Sci & $0.76^{\star \star}$ & $0.884^{\star \star}$ & $0.97^{\star \star}$ & $0.753^{\star \star}$ \\
\hline Agr Sci & $0.83^{\star \star}$ & 1.086 & 0.850 & 0.803 \\
\hline Ped & $0.97^{\star \star}$ & $1.034^{\star \star}$ & $0.893^{\star \star}$ & $0.825^{\star \star}$ \\
\hline Chem & $1.276^{\star \star}$ & $0.816^{*}$ & 0.775 & 0.631 \\
\hline Econ & $1.03^{\star \star}$ & $0.861^{\star \star}$ & $1.056^{\star \star}$ & $0.926^{\star \star}$ \\
\hline Phys & 1.288 & 1.062 & 1.167 & 0.919 \\
\hline Sociol & $1.063^{\star *}$ & $1.006^{\star \star}$ & 0.762 & $0.668^{*}$ \\
\hline Math & 1.749 & 1.007 & 0.733 & 0.827 \\
\hline Arts & $0.728^{*}$ & $0.759^{\star \star}$ & 0.808 & $0.724^{\star *}$ \\
\hline Earth Sci & 0.790 & 1.180 & 0.619 & 0.732 \\
\hline Ling & $1.096^{\star \star}$ & $1.624^{\star \star}$ & $1.193^{\star \star}$ & $0.838^{\star \star}$ \\
\hline Hist & $0.67^{\star}$ & $1.092^{\star \star}$ & 1.080 & $0.876^{\star}$ \\
\hline Polit & $0.738^{\star \star}$ & 0.660 & 0.962 & $0.724^{*}$ \\
\hline Law & $0.518^{\star \star}$ & $1.026^{\star \star}$ & $0.841^{\star \star}$ & $0.731^{\star \star}$ \\
\hline Psych & $1.08^{\star \star}$ & $1.152^{\star \star}$ & $1.23^{\star \star}$ & $0.986^{\star \star}$ \\
\hline Philo & $1.004^{\star \star}$ & 1.766 & 1.244 & 1.293 \\
\hline Anthro & 0.245 & $1.318^{\star \star}$ & 0.955 & 1.033 \\
\hline Geo & $0.825^{\star \star}$ & $1.025^{\star \star}$ & 0.981 & $0.853^{*}$ \\
\hline Astro & 0.000 & 0.366 & 0.803 & 0.673 \\
\hline Ethics & 1.222 & $0.354^{\star *}$ & $1.135^{\star \star}$ & $0.932^{\star \star}$ \\
\hline \multicolumn{5}{|c|}{$\begin{array}{l}\left({ }^{\star}\right) \text { Áreas en las que hay un mayor número de investigadoras. } \\
\left(^{\star \star}\right) \text { Áreas en las que hay un mayor número de investigadoras que producen más } \\
\text { publicaciones que sus compañeros investigadores. }\end{array}$} \\
\hline
\end{tabular}

Por último, a partir de un gráfico relacional se presentan en la Figura 3 las 10 áreas más productivas para cada uno de los tres niveles de las categorías profesionales de los investigadores. En el eje de abscisas se muestran los valores de GPI-r y en el de ordenadas los de GPI-p de las áreas seleccionadas. Además, se han marcado dos ejes en GPI=1 para diferenciar aquellas áreas en las que la presencia de la mujer está por encima o debajo de la de los hombres, y en la que su productividad es mayor o menor que la de sus compañeros varones. La línea 
de 45 grados actúa como una frontera de eficiencia de género de modo que aquellas áreas que están por encima de ella demuestran una mayor eficiencia de las mujeres, y en las que están por debajo de ella, la eficiencia mayor corresponde a los hombres. En la figura se observa que es el nivel de candidato $(\mathrm{Ca})$ donde las mujeres se muestran más eficientes, como ocurre en las áreas de Física (Phys), Matemáticas (Math), Química (Chem) y Sociología (Sociol). En el nivel L_1 son Pediatría (Ped), Ciencias Agrarias (Agr Sci) y Ciencias de la Tierra (Earth Sci) las áreas más eficientes desde el punto de vista de las mujeres. En los niveles más elevados (Up_ L), a excepción de la Física (Phys), que se muestra prácticamente en la línea de 45 grados, no hay ninguna área de conocimiento en la que las mujeres sean más eficientes que los hombres.

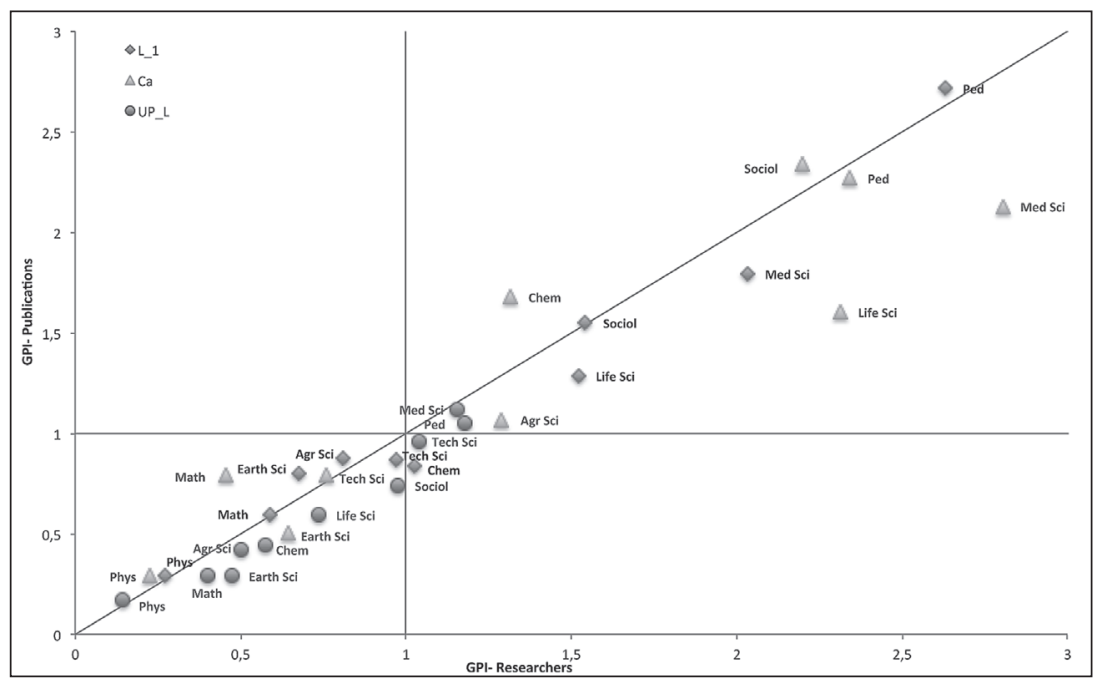

Figura 3. Gráfico relacional. 10 áreas más productivas (>3.000 publicaciones). Tres niveles profesionales.

\section{Conclusiones}

En términos generales, la investigación en el sistema científico venezolano recogida en el Programa de Promoción del Investigador se caracteriza por tener un mayor número de mujeres que de hombres en la actividad investigadora; sin embargo, en términos agregados la actividad científica de los hombres, medida por los artículos en revistas nacionales e internacionales, es superior a la de las mujeres. Cuando ambas distribuciones se analizan por área temática, no se observan diferencias significativas debidas al género ( $p$-value $>0.05)$. Desde el punto de vista del número de investigadores, entre 
las cinco áreas con mayor número de ellos, en tres se ha observado una fuerte presencia femenina, como en Ciencias de la Vida (Life Sci, 55.6\%), Ciencias Médicas (Med Sci, 65.9\%) y Pediatría (Ped, 68.6\%). También hay que destacar una mayor presencia de la mujer en otras áreas con menor número de investigadores como son las áreas de ciencias sociales, Economía (Econ, $60.7 \%$ ), Lingüística (Ling, $72.9 \%$ ), Derecho (Law, 68.8\%) y Psicología (Psych, $74.5 \%$ ).

Esta prevalencia de las mujeres también se mantiene al considerar las publicaciones que realizan en estas áreas mencionadas, a excepción de Ciencias de la Vida (Life Sci) en donde publican menos documentos que los hombres. Sin embargo, en términos generales los porcentajes de participación de las mujeres en la producción científica en estas áreas son inferiores al porcentaje esperado por su presencia respecto al número de investigadoras en ellas. Caputo, Requena y Vargas (2012) muestran una conclusión similar respecto a las Ciencias de la Vida (Life Sci) cuando afirman que "las biólogas venezolanas son menos productivas que sus colegas varones". Esto es un primer indicador de la menor eficiencia de la mujer en el sistema científico venezolano, que puede ser debido a las mayores dificultades que ha tenido la mujer para incorporarse plenamente a las actividades investigadoras y que es un fenómeno que ha sido observado en otros países (ETAN, 2000; Pérez Sedeño et al., 2003).

Si los datos analizados se desagregan por categoría profesional y por género, se observa a nivel global una menor presencia y eficiencia femeninas en la categoría superior (Up_L), respecto de los hombres. Cuando se considera el número de investigadores en las tres categorías profesionales de cada una de las áreas temáticas, las diferencias observadas no son estadísticamente significativas ( $\mathrm{p}>0.05$ ); sin embargo, al considerar el número de publicaciones resulta que sí lo son $(\mathrm{p}<0.01)$. Dado que la tendencia es que conforme se asciende en la categoría profesional las mujeres son menos productivas que los hombres, podemos afirmar que tal tendencia no se debe al azar sino a otras causas, en tanto que algunas de ellas ya han sido analizadas en otros trabajos de investigación, como por ejemplo las vinculadas con la edad (Fox, 1983), con la atención a los hijos (Prpić, 2002) o con el nivel de especialización que en determinados estudios se ha observado ser menor en el caso de las mujeres (Leahey, 2006).

Cuando se analizan los índices de paridad los resultados que se han obtenido son consistentes con los resultados comentados. Estas medidas nos permiten comparar el nivel de paridad que se da tanto entre los inputs (el número de investigadores) como en los outputs (el número de publicaciones). A nivel global, en medias ponderadas, la presencia de la mujer en el 
sistema científico venezolano es prácticamente un $32 \%$ superior a la de los hombres; sin embargo su productividad es casi un $5 \%$ inferior a la de éstos. Estas diferencias son más acusadas en el nivel superior de la categoría profesional (Up_L), donde el valor promedio del número de mujeres es un $17 \%$ menor que el de los hombres y donde ellas publican un $27 \%$ de documentos menos que los hombres. Sin embargo, aunque las diferencias observadas en las tres categorías profesionales son significativas, se podría decir que no son concluyentes, ya que el valor obtenido es muy próximo al valor crítico $(\mathrm{p}=0.046)$. Cuando se considera el número de publicaciones en las tres categorías profesionales las diferencias entre hombres y mujeres no son significativas $(\mathrm{p}>0.05)$.

El cálculo de la tasa de eficiencia en la productividad de las investigadoras venezolanas muestra que en valores globales las mujeres son ligeramente más eficientes que los hombres en tres de las áreas analizadas: Filosofía (Philo), Antropología (Anthro) y Ciencias Tecnológicas (Tech Sci)). Si consideramos la tasa de éxito de las mujeres por categoría profesional, se observa que no se puede vincular la paridad con la eficiencia, puesto que en situaciones de paridad favorables hacia la mujer su eficiencia puede ser mayor o menor que la de los hombres en cualquiera de las categorías profesionales; por ejemplo, en el caso de Ciencias de la Vida (Life Sci) en todas sus categorías profesionales, aun teniendo las mujeres una mayor presencia y mayor productividad que los hombres, ésta es menor que la esperada, lo que indica que los hombres aun teniendo un número menor de investigadores son más eficientes que las mujeres en su producción científica.

Cuando la paridad es desfavorable para las mujeres tanto en el número de investigadoras como en su producción científica, pueden mostrarse más eficientes que los hombres; esto se ha observado en todas las categorías profesionales en el área de Física (Phys), así como en el nivel de candidato (Ca) del área de Matemáticas (Math).

Sin embargo, en todas las situaciones en las que la mujer tiene una mayor presencia respecto a los hombres, pero donde su producción científica es inferior a la de éstos, se ha observado que la eficiencia de las mujeres siempre es menor que la de los hombres. Esto sucede en muy pocos casos, como en la categoría profesional L_1 en el área de Química (Chem) y en la categoría Up_L de Ciencias Tecnológicas (Tech Sci).

Los resultados de este estudio nos han permitido observar que la mayor presencia de la mujer en el ámbito científico no ha servido para mejorar su productividad. En este sentido las políticas encaminadas a aumentar la presencia de la mujer en la investigación no son suficientes, ya que los resultados obtenidos en este trabajo indicarían que las políticas de paridad por sí solas 
no implican necesariamente igualdad de oportunidades, aunque probablemente sean una condición necesaria para lograrlo. Tal y como se indica en el Informe ETAN (2000), existen un gran número de condicionantes (sociales, económicas y políticas) que podrían explicar los resultados observados en este estudio, en tanto que afectan a la carrera científica de las mujeres, y no son fáciles de solucionar en el corto periodo de tiempo que ha pasado desde que se han empezado a implementar acciones desde distintas perspectivas para corregir esta injusta situación.

\section{Bibliografía}

Abramo, G.; D’Angelo, C.A.; Caprasecca, A. (2009), "Gender differences in research productivity: A bibliometric analysis of the Italian academic system", en Scientometrics, 79 (3): 517-539.

Allison, P. D. (1978), "Measures of inequality", en American Sociological Review, pp. 865-880.

Cañibano, C.; Otamendi, J.; Andújar, I. (2008), "Measuring and assessing researcher mobility from $\mathrm{CV}$ analysis: the case of the Ramón y Cajal Programme in Spain”, en Research Evaluation, 17 (1): $17-31$.

Caputo, C.; Requena, J.; Vargas, D. (2012), "Life sciences research in Venezuela", en Scientometrics, 90 (3): 781-205.

De Filippo, D.; Sanz-Casado, E.; Gómez, I. (2009), "Movilidad científica y género: Estudio del profesorado de una universidad española", en Revista Mexicana de Sociología, 71 (2):351-396.

Delgado de Smith, Y.; Rojas Martini, M. (2009), "Mujeres en la ciencia: referencias mundiales y locales", en Y. Delgado de Smith \& M. C. González (eds.), Mujeres en el Mundo: Ciencia, género, migraciones, arte, lenguaje y familia, Valencia, Venezuela: Laboratorio de Investigación en Estudios del Trabajo (LAINET), pp. 37-61, disponible en: http://www.claudiahasanbegovic.com.ar/publica ciones/42.pdf\#page $=37$ (Fecha de consulta: 12 de septiembre del 2013).

ETAN (2000), Science Policies in the European Union: Promoting Excellence through Mainstreaming Gender Equality. A Report from the ETAN Expert Working Group on Women and Science, Luxembourg: Office for Official Publications of the European Communities.

Fox, M. F. (1983), "Publication Productivity among Scientists: A Critical Review", en Social Studies of Science, 13 (2):285-305.

García González, P. E. (2010), Diseño, desarrollo y aplicación de un método para el análisis y tratamiento de la información con fines métricos [Tesis Doctoral], Madrid: Universidad Carlos III de Madrid. 
Gaughan, M.; Bozeman, B. (2002), "Using curriculum vitae to compare some impacts of NSF research grants with research center funding", en Research Evaluation, 11 (1): 17-26.

Leahey, E. (2006), "Gender differences in productivity”, en Gender E Society, 20 (6): 754-780.

Marcano, D.; Phélan, M. (2009), "Evolución y desarrollo del programa de promoción del investigador en Venezuela", en Interciencia, 34 (1): 17-24.

Martín-Sempere, M. J.; Rey-Rocha, J. (2003), Evaluación y Seguimiento De Programas De Movilidad Del Personal Investigador y Del Profesorado Universitario. Informe Del Proyecto EA 2003-0028, Madrid: Ministerio de Educación.

Mauleón, E.; Bordons, M. (2006), "Productivity, impact and publication habits by gender in the area of Materials Science”, en Scientometrics, 66 (1): 199-218.

—_ Oppenheim, C. (2008), "The effect of gender on research staff success in life sciences in the Spanish National Research Council”, en Research Evaluation, 17 (3): 213-225.

Meyer, D.; Zeileis, A.; Hornik, K. (2006), “The strucplot framework: Visualizing multi-way contingency tables with vcd", en Journal of Statistical Software, 17 (3), disponible en: http://www.jstatsoft. org/v17/i03/paper (Fecha de consulta: 12 de septiembre del 2013). ; Friendly, M. (2012), Visualizing Categorical Data. R Package Version 1.2-12, disponible en: http://cran.r-project.org/web/packa ges/vcd/vcd.pdf (Fecha de consulta: 12 de septiembre del 2013).

ONCTI Observatorio Nacional de Ciencia, Tecnología e Innovación (2007), Caracas, Venezuela, página web: www.oncti.gob.ve (Fecha de consulta: 12 de septiembre del 2013).

Pérez Sedeño, E. et al. (2003), La situación de las mujeres en el sistema educativo de ciencia y tecnología en España y su contexto internacional, Madrid: MEC (Programa de Análisis y Estudios de Acciones Destinadas a la Mejora de la Calidad de la Enseñanza Superior y de Actividades del Profesorado Universitario, REF: S2/ EA2003-0031), disponible en: http://www.oei.es/salactsi/EA2003 -0031.pdf (Fecha de consulta: 12 de septiembre del 2013).

Prozesky, H. (2008), “A Career-History Analysis of Gender Differences in Publication Productivity among South African Academics", en Science Studies, 21 (2): 47-67.

_ (2006), "Gender differences in the journal publication productivity of South African academic authors", en South African Review of Sociology, 37 (2): 87-112.

Prpić, K. (2002), "Gender and productivity differentials in science", en Scientometrics, 55 (1): 27-58.

R Development Core Team (2010), A Language and Environment for Statistical Computing, Vienna, Austria: R Foundation for Statistical Computing, disponible en: http://www.R-project.org (Fecha de consulta: 12 de septiembre del 2013). 
Rousseau, R. (1998), "Evenness as a descriptive parameter for department or faculty evaluation studies", en E. de Smet (ed.), Informatiewetenschap 1998, Antwerp, Belgium: Werkgemeenschap Informatiewetenschap, pp. 135-145.

- (2000), "Concentration and evenness measures as macro-level scientometric indicators", en Second International Seminar on Quantitative Evaluation of Research Performance, Shanghai, 2325 October, disponible en: http://users.pandora.be/ronald.rousseau/Rousseau_Shanghai_2000.pdf (Fecha de consulta: 12 de septiembre del 2013).

Sandström, U. (2009), "Combining curriculum vitae and bibliometric analysis: mobility, gender and research performance", en Research Evaluation, 18 (2): 135-142.

UNESCO (1997), Gender-sensitive education statistics and indicators. A practical guide, París: UNESCO, Division of statistics.

República de Venezuela (1990), Decreto núm. 928, del 7 de junio de 1990, en Gaceta Oficial, 34.486, 11 de junio.

Zeileis, A. (2012), Ineq: Measuring Inequality, Concentration, and Poverty, R package version 0.2-10, disponible en: http://cran.r-project .org/web/packages/ineq/ineq.pdf (Fecha de consulta: 12 de septiembre del 2013). 